Necesidades de formación docente de los maestros de

Educación Artística a nivel Secundaria del Instituto de Educación de Aguascalientes'

Pedagogycal weaknesses in the formation of arteducation teachers at middleschool level at the Education Institute of Aguascalientes

RAÚL W. CAPISTRÁN GRACIA raul_capistran@hotmail.com

Departamento de Música

Universidad Autónoma de Aguascalientes

\section{Educación y Pedagogía}

$\Rightarrow$ Recibido 02/08/2017

$\checkmark$ Aceptado 15/10/2017

\section{Resumen}

Gracias a la Reforma Integral de la Educación Básica (RIEB) la educación artística en México se convirtió, en el año 2006, en una asignatura obligatoria-formativa dentro del plan de estudios (SEP, 2006a). Para implementar exitosamente las reformas educativas, el profesor debe poseer conocimientos disciplinares sólidos, así como fundamentos teórico-pedagógicos que le permitan organizar y dirigir un proceso de enseñanza y aprendizaje integral. Este artículo presenta los resultados más relevantes de una investigación de tipo exploratorio y corte mixto cuyo objetivo consistió en determinar las necesidades de formación docente de los maestros de educación artística, del Instituto de Educación de Aguascalientes (IEA) a fin de proponer y promover medidas de mejora. Tomando algunos de los indicadores propuestos por Shulman (2005) y sobre la base de los objetivos de los programas de materia, se creó un cuestionario por cada disciplina artística. En la encuesta participaron 103 maestros.

Los resultados indican que muchos de ellos poseen una formación disciplinar sólida. Sin embargo, también se detectaron deficiencias importantes. Muchos carecen de estudios profesionales en las artes, desconocen las propuestas metodológicas más importantes en educación artística y existe falta de participación en cursos de formación continua. Finalmente, algunos maestros desconocen la existencia de los materiales didácticos que proporciona la Secretaría de Educación Pública. Por lo anterior, es muy importante que el IEA mantenga un diálogo abierto con sus maestros, favorezca la profesionalización de los docentes y promueva la impartición de cursos de educación continua y talleres que le permitan mantenerse actualizado y conocer los materiales a su disposición.

\section{Palabras clave \\ Educación artística · Educación continua · Pedagogía · Debilidades y fortalezas}

1 La investigación cuyos resultados se presentan en este artículo fue patrocinada por la Dirección General de Investigación y Posgrado de la Universidad Autónoma de Aguascalientes. 


\section{Introducción}

La educación artística representa un ámbito general de educación, ya que contribuye al desarrollo de valores formativos generales y desarrolla competencias que implican destrezas, hábitos, actitudes y conocimientos que constituyen herramientas que ayudan a los educandos a decidir y realizar su proyecto de vida (Touriñán, 2006). En México, la Refor ma Integral de la Educación Básica (RIEB) a través de su Plan de Estudios 2011 (SEP, 2011a), se orienta a propiciar el desarrollo y fortalecimiento de las competencia artísticas. Para poder enfrentar el reto de proporcionar una educación artística de calidad, se deben tomar en cuentas algunas variables y condicionantes. Para comenzar, en la actualidad, el arte no es considerado un don divino accesible a unos cuantos. Por el contrario, se considera que la construcción del conocimiento artístico depende, al igual que cualquier otra disciplina, de un proceso de enseñanza y aprendizaje pedagógicamente estructurado (Pimentel, Coutinho y Guimarães, 2011).

Por lo anterior, el maestro debe posee una formación disciplinar de alto nivel, que vaya acompañada de conocimien- tos teórico-pedagógicos sólidos. En ese mismo sentido, las instituciones deben promover la actualización de los conocimientos de los maestros a través de procesos de formación continua, de tal manera que los maestros puedan adaptarse al cambiante mundo de la educación (Giráldez, 2011).

Finalmente, para impartir educación artística con seriedad, se requieren maes tros sensibles; profesores competentes que transmitan no solo los conocimientos disciplinares y coordinen las acciones durante la clase, sino que también sean capaces de manejar sabiamente elementos subjetivos como las emociones, la sensibilidad y el sentido de identidad (Pimentel, Coutinho y Guimarães, 2011) y los canalicen hacia la creatividad, y la originalidad. Uno de los grandes retos que enfrenta la educación artística, está representado por la brecha existente entre la formación del profesorado, fundamentada principalmente en la producción artística, y su dominio docente. En otras palabras, en muchas ocasiones, los maestros poseen la técnica para crear, pero no para transmitir de manera pedagógica y sensible su saber (Martínez, 2010). En ese sentido, es imperativo que los maestros se formen en el ámbito de la educación 
artística de manera integral, mediante carreras y licenciaturas especializadas que enfaticen tanto los ideales pedagógicas como el rigor disciplinar, para que puedan cumplir con calidad y eficiencia su labor docente (Yohary y Araujo, 2014).

En conclusión, es imperativo contar con profesores altamente capacitados para impartir la asignatura de educación artística y cumplir con los estándares necesarios; con maestros que puedan desempeñar no solo el papel de pedagogo-artista, sino también el de artista-pedagogo, como lo espera la Secretaria de Educación Pública de México (SEP, 2011c). Sin embargo, no se pueden "borrar de un plumazo" las deficiencias que históricamente se vienen arrastrando y quedan muchas áreas de oportunidad por atender. Para empezar, en México, las carreras en artes con reconocimiento de validez oficial son sumamente recientes. Por dar uno, de entre varios ejemplos, la primera licenciatura en música fue ofrecida en 1968 por la Universidad Nacional $\infty \quad$ Autónoma de México. Fue 41 años desㄱ pués (2009) que la Universidad AutónoD. ma de Aguascalientes ofreció una licenI ciatura en música y todavía tuvieron que - pasar cinco años más, para que egresara la primera generación. Si a lo anterior se añade, que las primeras carreras en artes enfatizaban la ejecución o la producción, puede concluirse que, en el mejor de los casos, cuando un licenciado especializado en alguna de las disciplinas artísticas tomaba una plaza de maestro, la mayor parte del tiempo carecía de los conocimientos didáctico-pedagógicos para impartir sus clases (Ávila, 2013). Es necesario añadir que, hasta la fecha, México cuenta con muy pocas instituciones que ofrezcan carreras en artes (Autor, 2016). Como consecuencia, hasta el año 2014, el perfil mínimo de contratación requerido por la Secretaria de Educación Pública, indicaba que bastaba contar con un bachillerato y constancia de estudios artísticos para ser contratado (SEP, 2014).

Por todo lo expuesto, la investigación, cuyos resultados se presentan a continuación, tuvo como objetivo determinar las necesidades de formación docente de los maestros de educación artística a nivel secundaria del Instituto de Educación de Aguascalientes, ${ }^{2}$ con el propósito de proponer y promover medidas de mejora a través de futuras intervenciones.

2 El Instituto de Educación de Aguascalientes es una dependencia gubernamental encargada de proporcionar educación gratuita de carácter obligatorio a los habitantes del estado de Aguascalientes, México.

\section{Problema de investigación}

Debido a los antecedentes antes ex puestos, se desconocen las necesidades de formación docente de los maestros de artes a nivel de educación secundaria en el estado de Aguascalientes, México, lo que impide hacer propuestas de mejora realistas que tengan un impacto certero en los procesos de enseñanza y aprendizaje de las artes.

\section{Objetivo General}

Obtener a través de la técnica de la encuesta, información cuantitativa $y$ cualitativa, relativa a las necesidades de formación docente de los maestros de educación artística del IEA (música, danza, teatro y artes plásticas) a nivel secundaria.

\section{Objetivos específicos}

a) Identificar el nivel académico general del maestro de educación artística;

b) Determinar su nivel de conocimientos disciplinares; c) Determinar el nivel de conocimientos en educación artística y e) Identificar las áreas fuertes y de oportunidad que existen en la preparación de los docentes especializados en educación artística.

\section{Marco teórico}

De acuerdo con Shulman (2005) la complejidad del conocimiento del profesor demanda el establecimiento de categorías de análisis del mismo. Shulman considera que ese conocimiento, deberían incluir por lo menos las siguientes siete categorías:

1. Conocimiento del contenido;

2. Conocimiento didáctico general, teniendo en cuenta especialmente aquellos principios y estrategias generales de manejo y organización de la clase que trascienden el ámbito de la asignatura;

3. Conocimiento del currículo, con un especial dominio de los materiales y los programas que sirven como "herramientas para el oficio" del docente; 
4. Conocimiento didáctico del contenido: esa especial amalgama entre materia y pedagogía que constituye una esfera exclusiva de los maestros, su propia forma especial de comprensión profesional;

5. Conocimiento de los alumnos y de sus características;

6. Conocimiento de los contextos educativos, que abarcan desde el funcionamiento del grupo o de la clase la gestión y financiación de los distritos escolares, hasta el carácter de las comunidades y culturas; $y$

7. Conocimiento de los objetivos, las finalidades y los valores educativos, y de sus fundamentos filosóficos e históricos (Shulman, 2005, p. 11).

Shulman (2005) advierte que, el hecho de centrarse en el conocimiento de maestro, no implica que él, sea el actor más importante del proceso de enseñanza y aprendizaje. Por el contrario, este autor admite la importancia de la educa@ ción centrada en el estudiante, pero afirma: "incluso en las formas de educación más centradas en el alumno, donde gran parte de la iniciativa está en manos de los estudiantes, apenas hay margen para la ignorancia del maestro" (p. 10)

La investigación cuyos resultados se comparten en este reporte, se centra en las necesidades de formación docente, a fin de proponer y promover mejoras a través de futuras intervenciones. En ese sentido, se toman los siguientes puntos o indicadores propuestos por Shulman: "conocimiento del contenido", "conocimiento del currículo", "conocimiento didáctico del contenido", "conocimiento de los objetivos", "las finalidades y los valores educativos", y conocimiento de sus "fundamentos filosóficos e históricos" (Shulman, 2005, p. 11).

\section{Metodología}

La Investigación fue de tipo exploratorio y de corte mixto. Los cuestionarios incluyeron preguntas contextuales, preguntas con escalas de medición Likert y preguntas abiertas. Para determinar las áreas de oportunidad en la formación docente de los maestros de cada disciplina, se crearon cuatro escalas o cuestionarios con la ayuda de cuatro especialistas, quienes diseñaron los ítems sobre la base de los objetivos generales del Programa de Estudios 2011, Guía Para el Maestro, Educación Básica Secundaria, Artes, 2011 (SEP, 2011 b, p. 25, 39, 53, 67). Para validar la es- cala se utilizó la técnica de juicio de expertos, en la que participaron cuatro profesores especializados en cada disciplina artística. Tres de las escalas obtuvieron coeficientes altos de confiabilidad interna (música, $a=.896$; teatro: $\alpha=.907$, artes visuales: $\mathrm{a}=.921$ ) mientras que el cuestionario para los maestros de expresión corporal y danza obtuvo un coeficiente bajo: $a=.561)$.

Los ítems representaron conocimientos prácticos y teórico-metodológicos indispensables para llevar a cabo la labor docente. El maestro debía circular o marcar con una " $x$ " el descriptor que mejor reflejara su nivel de conocimientos o habilidad artística específica: "inexistentes", "limitados", "suficientes", "buenos" y "muy buenos". Para propósito de análisis, se determinó considerar cono fortaleza aquellos reactivos/conocimientos que obtuvieran mayoritariamente respuestas de "muy buenos" y "buenos" y considerar como áreas de oportunidad aquellos reactivos/conocimientos cuyas respuestas fueran mayoritariamente "suficientes", "limitados" e "inexistentes", pues, aun cuando poseer un conocimiento suficiente, representa el mínimo para desempeñar la labor docente, no constituye el ideal en la preparación de un maestro, sino que se debe procurar que los maestros tengan un conocimiento disciplinar sólido, que los habilite para resolver de manera eficiente los retos que implica la impartición de su asignatura (Pimentel, Coutinho y Guimarães, 2011; Giráldez, 2011; Yohary y Araujo, 2014).

Los maestros fueron convocados por el propio IEA, los días 26 de febrero y 21 de abril de 2016. El cuestionario fue aplicado por el propio investigador. El llenado de los cuestionarios fue voluntario y anónimo. Los datos fueron capturados, procesados y analizados con la ayuda de programa Statistics Package for the Social Sciences IBM SPSS 2.0

\section{Resultados y discusión}

En el estudio, participaron 103 maes tros de artes a nivel de educación secundaria del Instituto de Educación de Aguascalientes (IEA) de un total de 128 (Música, 26; Teatro, 9; Exp. Corporal y Danza, 48 y Artes Visuales, 20). La muestra tomada fue de oportunidad o conveniencia (Creswell, 2001). Sin embargo, un cálculo estadístico posterior reveló que la muestra tendría un 5\% de margen de error y un $95 \%$ de confiabilidad. De los participan- 
tes, 44 fueron hombres (42.7\%) y 59 fueron mujeres (57.3\%). Sus edades oscilaron entre los 22 y los 55 años con una media de 38.67 y una DS de 8.828. En lo que se refiere al grado académico, los números más preocupantes, están representados por dos maestros que solo cuentan con secundaria y siete con preparatoria. 18 de los profesores cuenta con una carrera técnica y tres con un bachillerato en música sacra, el cual, carece de validez oficial.

Las licenciaturas que los encuestados dijeron poseer, fueron clasificadas en tres:

a) Licenciatura disciplinar: Aquellas que están íntimamente relacionadas con la asignatura a enseñar. Por ejemplo, si la asignatura es música, la licenciatura disciplinar correspondiente será la de licenciado en educación musical o licenciado en música.

b) Licenciatura artística no disciplinar: Aquellas licenciaturas en educación artística o en alguna otra disciplina artística que suponen una preparación menos especializada. Por ejemplo, en teatro encontramos a un licenciado en letras hispánicas. Aun cuando su preparación podría habilitarlo para impartir la clase, su nivel de especialización no es tan alto como la del licenciado en artes escénicas o el licenciado en teatro.

c) Licenciatura no disciplinar: Aquellas licenciaturas totalmente ajenas a la disciplina que imparten. Por ejemplo, un ingeniero industrial impartiendo danza.

De los 103 maestros encuestados, 25 son licenciados especializados en la asignatura que enseñan, 14 son licenciados en otras disciplinas artísticas y 34 tienen una preparación profesional ajena a la disciplina que imparten, lo que refleja el grado de laxitud que existía en los perfiles de contratación. Como se mencionó anteriormente, bastaba con poseer, por lo menos, un certificado de bachillerato y constancias de estudios artísticos para ser candidato a una plaza de maestro de educación artística.

\begin{tabular}{|c|c|c|c|c|c|c|c|c|}
\hline $\begin{array}{l}\text { Disciplina } \\
\text { Artística }\end{array}$ & 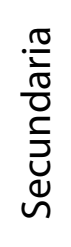 & 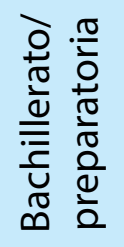 & 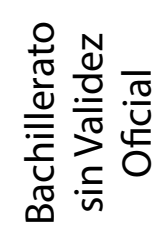 & 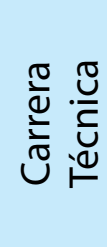 & 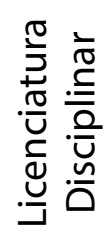 & 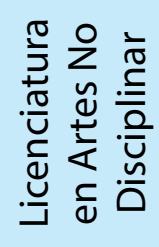 & 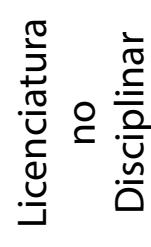 & ڤँ \\
\hline Música & 1 & 5 & 3 & 4 & 4 & 1 & 8 & 26 \\
\hline Teatro & 0 & 0 & 0 & 0 & 6 & 2 & 1 & 9 \\
\hline $\begin{array}{l}\text { Artes } \\
\text { Visuales } \\
\end{array}$ & 0 & 0 & 0 & 0 & 4 & 8 & 8 & 20 \\
\hline $\begin{array}{l}\text { Exp. Corp, y } \\
\text { Danza }\end{array}$ & 1 & 2 & 0 & 14 & 11 & 3 & 17 & 48 \\
\hline Total & 2 & 7 & 3 & 18 & 25 & 14 & 34 & \\
\hline
\end{tabular}

Tabla 1. Nivel académico de los profesores por disciplina.

Uno de los objetivos de la educación artística es lograr una formación integral que permita que todos los estudiantes reciban una educación apropiada que les permita alcanzar los mejores resultados posibles de acuerdo con cada nivel de desarrollo. Por lo anterior, es vital que el profesor no solo posea conocimientos disciplinares prácticos, sino también fundamentos teórico-pedagógicos que le permitan organizar y dirigir un proceso de enseñanza aprendizaje que fomente el desarrollo de los procesos lógicos del pensamiento para que, a medida en que el estudiante se apropie de conocimientos y procedimientos, se eleva su capacidad de resolver problemas.

Debido a la falta de profesionalización que aún existe en la enseñanza de las artes, muchos profesores suelen fundamentar su labor docente en los conocimientos prácticos que poseen y en su experiencia, más que en conocimientos teórico-metodológicos de educación artística. Por lo anterior, cada uno de los cuestionarios incluyó ítems para determinar el nivel de conocimientos disciplinares a nivel práctico, así como ítems para determinar el nivel de conocimientos teórico-metodológicos.

En la tabla 1, puede notarse que, proporcionalmente, los maestros de teatro son los que más licenciaturas disciplinares poseen (66.66\%), mientras que las otras disciplinas artísticas obtuvieron porcentajes muy bajos (música, 15.38\%; artes visuales, 20\% y Expresión Corporal y Danza, 22.91\%). Así, los resultados, tanto en conocimientos prácti- 
cos como en conocimientos teórico-metodológicos se correlacionan, en menor o mayor medida, con el nivel académico y sobre todo con el nivel de especialización.

La siguiente gráfica comparativa muestra las medias estadísticas obtenidas por los maestros en los ámbitos de conocimientos prácticos y conocimientos teórico-metodológicos. En ella, puede observarse que, con excepción de los maestros de teatro, quienes en la mayoría poseen carreras en la disciplina que imparten, existe una diferencia significativa entre los dos ámbitos en cada disciplina artística. También puede observarse que, de las cuatro disciplinas, los maestros con mayores necesidades docentes son los de música y artes visuales mientras que los maestros de teatro, obtuvieron la media más alta.

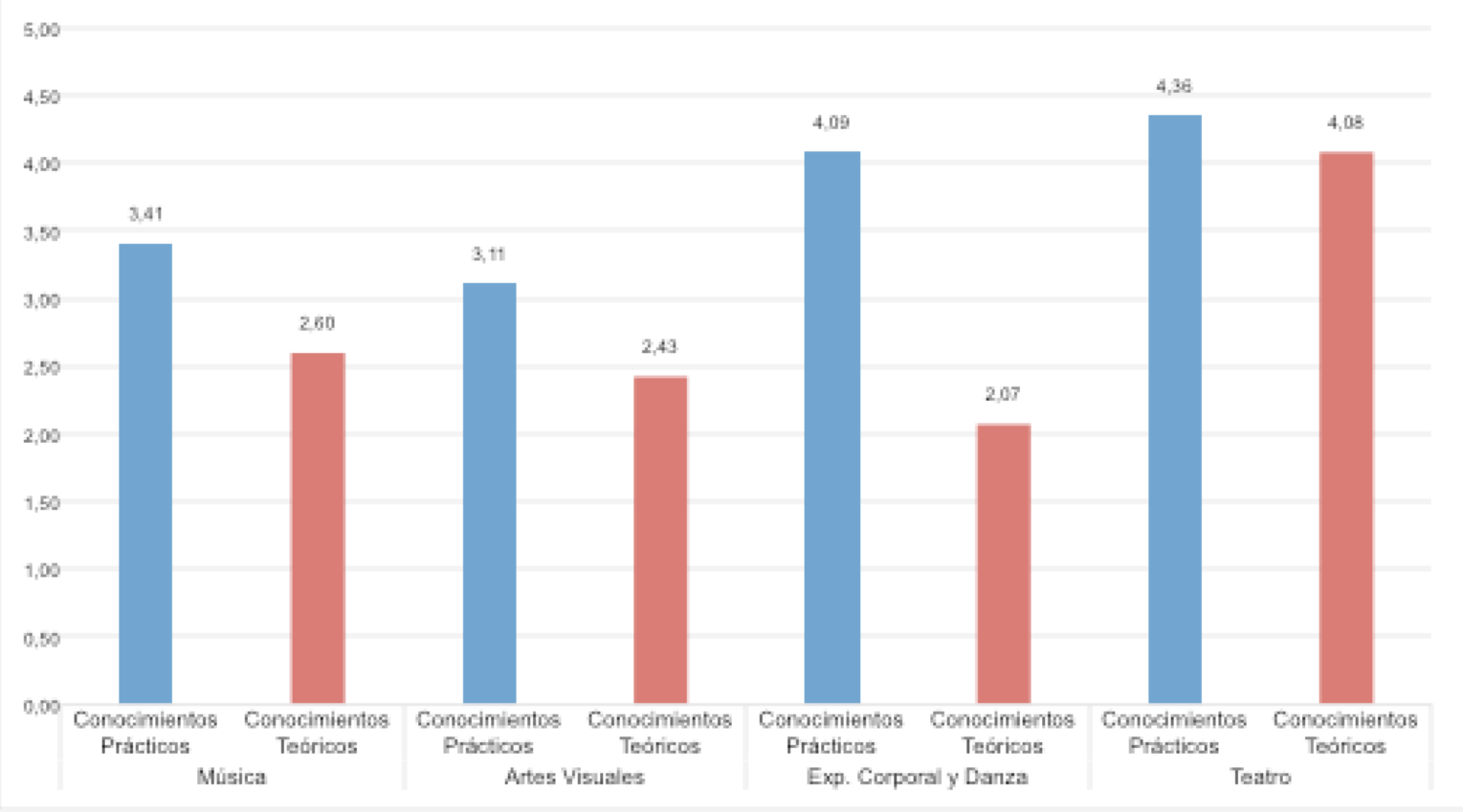

Gráfica 1. Diferencia entre la media del conocimiento de los profesores en el ámbito práctico y el ámbito profesional de cada disciplina artística.

En la Gráfica 1, destaca que, pesar del bajo porcentaje de profesores con licenciaturas disciplinares, los maestros de Expresión Corporal y Danza obtuvieron en conocimientos prácticos una media casi tan alta como la de los maestros de Teatro. Sin embargo, la media obtenida en conocimientos teóricos fue la más baja. Lo anterior podría deberse a la difusión y promoción que la práctica de la danza, como actividad extraescolar, ha tenido en México desde siempre.

20 Educación y Pedagogía
Los cursos, seminarios y talleres actualizan y preparan al maestro para una mejor impartición de su materia además de promover su sentido de pertenencia (Harwell, 2003). Sin embargo, existe un área de oportunidad de vital importancia en este ámbito, pues, un $46.75 \%$ de los maestros dice nunca asistir a cursos; un $36.36 \%$ dice hacerlo una vez al año y un $9.09 \%$ afirmar hacerlos dos veces año.

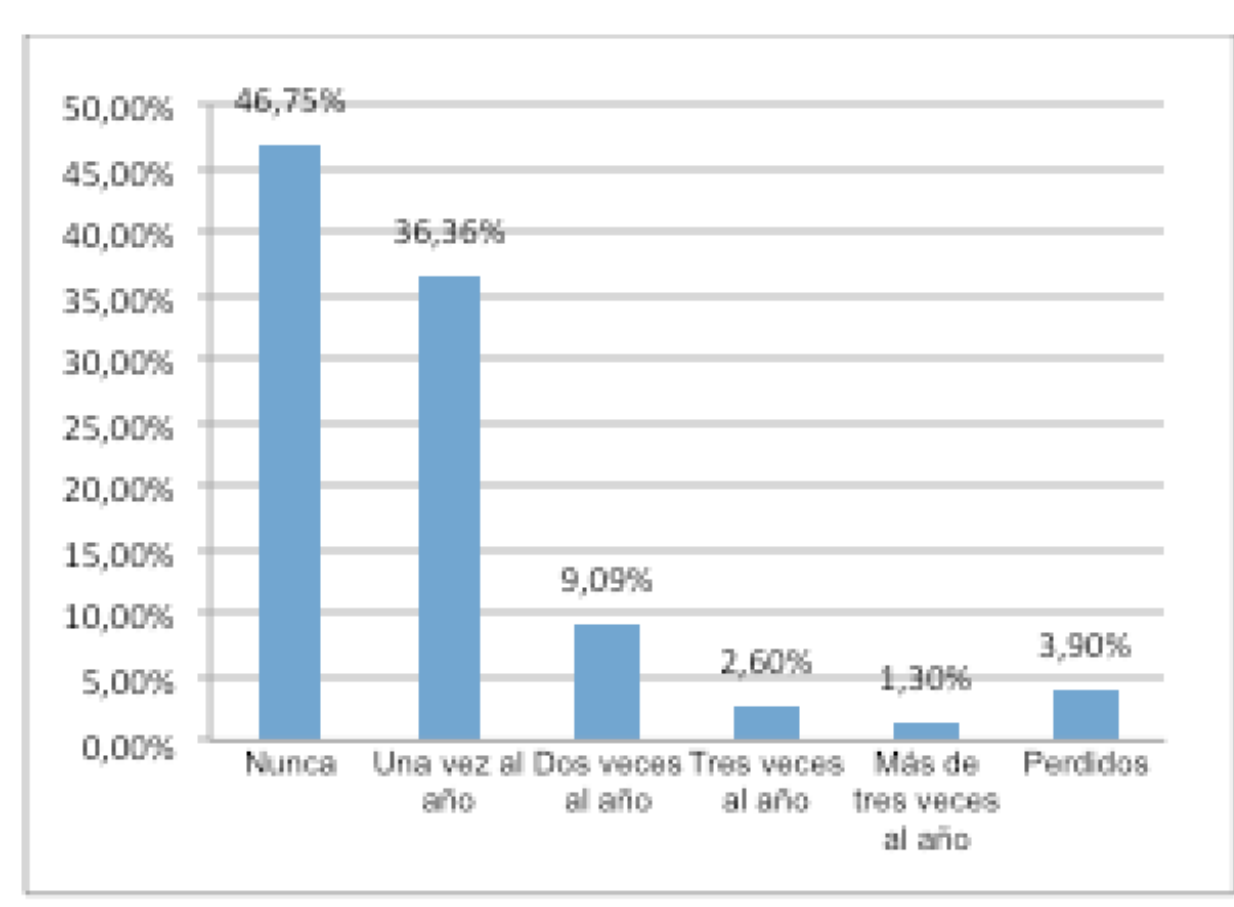

Gráfica 2. Número de veces al año que los maestros de secundaria reciben cursos y talleres de capacitación.

\section{Preguntas abiertas}

La Secretaria de Educación Pública ha puesto al alcance de los maestros de educación artística a nivel secundaria, algunos materiales básicos para que puedan llevar a cabo su labor docente. Entre los materiales que el maestro puede tener acceso a través de varias páginas de internet, se encuentran los libros Programas de Estudio 2011 Guía para el Maestro, Educación Básica, Secundaria (SEP, 2011b); Las Artes y su Enseñanza en la Educación Básica (SEP, 2011c); Artes. Guía de trabajo. Primer Taller de Actualización sobre los Programas de Estudio 2006 (SEP, 2006b) y Curso: Introducción a la educación Artística en el Contexto Escolar (SEP, 2009). Se espera que los maestros estén más que familiarizados con estos materiales, ya que, el primero, incluye los programas de ma- 
teria, a partir del cual deberá diseñar sus clases mientras que los demás, se constituyen como una fuente de información y consulta básica. Por todo lo anterior, se incluyó la pregunta “¿Cuál es su opinión respecto a los programas y materiales de apoyo para la asignatura de Artes de la SEP?" Las respuestas proporcionadas po los participantes fueron muy variadas, por lo que, para propósito del estudio, fueron conceptualizadas, categorizadas en unidades de análisis, estructuradas y organizadas siguiendo el modelo propuesto por González (1999).

Con respecto al programa de materia 18 de los participantes dieron opiniones positivas que estuvieron relacionadas con la utilidad del programa para guiar la labor docente. Algunas respuestas fueron: "En el programa está lo que debemos enseñar a los alumnos", "El programa abarca lo que los alumnos deben conocer y aprender", "El programa te guía y es el único programa permitido" y "Es muy importante todo lo que los programas proponen y muy indispensable para las clases", entre otras respuestas similares.

Por otro lado, un número importante de participantes, emitieron opiniones negativas del programa, relacionadas con la insuficiencia de la información (13 opiniones), la vaguedad en la redacción (6 opiniones), su simpleza (4 opiniones), la falta de apego a la realidad (2 opiniones), la poca utilidad del mismo (1 opinión) y la falta de congruencia (1 opinión). Entre esas opiniones se encontraron: “Creo que está muy ambiguo", "El programa es repetitivo y omite temas en algunos casos", “Está muy generalizado el contenido" "Mucha de la información no tiene mucha utilidad en muchos contextos" $y$ "Es necesaria más información".

Entre las opiniones más importantes proporcionadas por los maestros, se encontró la falta de libros de texto que, al igual que en el nivel de educación primaria, los ayuden a impartir la asignatura de arte. Entre las 28 opiniones vertidas, se encontraron las siguientes: "No hay libros para los alumnos por parte de la SEP", "No existen libros de texto a nivel secundaria por parte de la SEP", "La SEP no proporciona libros de texto y el contexto donde trabajamos son de baja solvencia económica", "Al igual que los libros de texto de otras asignaturas, a los alumnos se les debe entregar libro de texto" y "El simple hecho de que no hay libros brindados por la SEP para nuestros alumnos. Tienen que comprarlos y algunos directores no lo permiten". La espontaneidad en la respuesta de los maestros, respecto a esta área de oportunidad, parece estar conectada o relacionada con una de las premisas de esta investigación respecto a que, aun cuando ha habido avances importantes en materia de educación artística, aún queda mucho por hacer. El hecho de que la asignatura de Artes, sea una de las que no recibe libros de texto gratuitos, invita a reflexionar si las autoridades educativas siguen creyendo que esta asignatura no reviste la misma importancia que las demás.

Finalmente, se encontraron opiniones referentes tanto a la inexistencia de materiales didácticos (8 opiniones) como a la necesidad de contar con más materiales de apoyo (7 opiniones). Preocupa particularmente que algunos maestros ignoren la existencia de los materiales didácticos mencionados al principio de este apartado, pues se encontraron opiniones como:"ni siquiera sé si existen materiales didácticos para mi clase" o "no existen materiales didácticos para las artes". Por otra parte, es evidente la necesidad de proporcionar más materiales didácticos a los maestros, sobre todo, si se tiene en cuenta la amplitud y diversidad de cada disciplina artística.

\begin{tabular}{|l|c|}
\hline \multicolumn{1}{|c|}{ Categoría } & Frecuencias \\
\hline Carencia de libros de texto & 28 \\
\hline Opiniones positivas en cuanto a los programas & 19 \\
\hline Los programas son limitados, la información no es suficiente & 13 \\
\hline No tenemos materiales a nuestra disposición & 8 \\
\hline Necesidad de más materiales de apoyo & 7 \\
\hline Los programas son ambiguos & 6 \\
\hline Los programas son demasiado simples & 4 \\
\hline Los programas son aburridos & 2 \\
\hline Los programas no son realistas & 2 \\
\hline Los programas no proporcionan actividades & 1 \\
\hline Los programas son incongruentes & 1 \\
\hline Los programas no son de utilidad & 1 \\
\hline Muy generalizados & 1 \\
\hline Muy teóricos & 1 \\
\hline Muy repetitivos & 1 \\
\hline No contestaron & 9 \\
\hline
\end{tabular}

Tabla 6. Opiniones de los maestros respecto a los programas y materiales de apoyo de la SEP para la asignatura de Artes. 
La segunda pregunta abierta fue " ¿Por qué o porque no, considera usted suficiente el tiempo que se le dedica a la educación artística a nivel secundaria en México?" Cuatro (3.9\%) de los participantes, afirmaron que, si era suficiente, 97 (95.1\%) señalaron que no era suficiente $y$ dos se abstuvieron de responder (1\%). Desde e punto de vista del investigador, la mayoría de las razones que los profesores proporcionaron en relación al poco tiempo que se asigna a la asignatura de Artes, se apegan a las demandas que tantos pedagogos de las artes han hecho. En primer lugar, con 38 frecuencias, los maestros señalan que dos horas no eran suficientes para cubrir el programa de materia y llevar a cabo las actividades propias de cada disciplina. Entre las opiniones proporcionadas se encuentran "Son muchos los temas de programa y poco el tiempo de clase”, “No se cumplen los objetivos. Es muy poco tiempo y quedan inconclusos los temas", "2 horas a la semana resultan insuficientes para cubrir el programa" y "Los programas son muy amplios".

Un aspecto importante a destacar de las opiniones anteriores, es que, en la primera pregunta abierta, algunos los maes tros se quejaban de que los programas eran limitados, de que la información era insuficiente y ambigua y de que eran demasiado simples, aburridos y no realistas. Queda esta discrepancia para otra investigación que se enfoque específicamente en el contenido curricular de los programas.

En segundo lugar, con una frecuencia de 22 respuestas, se encontraron opiniones carentes de justificación que solo indicaban que la cantidad de tiempo para impartir la asignatura de Artes era muy poca. Opiniones como: "Dos horas no son suficientes", "Se necesita más tiempo", "Son únicamente dos sesiones semanales", "100 minutos a la semana son muy pocos" y "Se requieren mínimo tres horas por semana", invitan a reflexionar en la posibilidad de que el maestro no sepa con claridad porque no está de acuerdo en la cantidad de tiempo que se asigna a esta materia.

La tercera categoría se relaciona con la importancia que tiene la asignatura en la formación humanística del estudiante. 14 profesores vertieron opiniones tales como:"Se le debe dar más prioridad y más tiempo para una mejor formación de vida en el alumno", "La educación artística sensibiliza a la gente", "Las artes son parte del ser humano, como tal debe extenderse el tiempo" y "Las artes son necesarias para un buen desarrollo y crecimiento intelectual del alumno y solo se trabajan dos horas a la semana". Esta información es importante, pues indica una preocupación por parte del maestro que va más allá de la transmisión de conocimientos meramente técnico-artísticos y que repercute en la calidad de vida y en la construcción de actitudes positivas.

La cuarta categoría tiene que ver con el descontento que algunos profesores experimentan debido al poco valor que se le otorga a la asignatura de Artes. Esta categoría recibió 11 frecuencias. Entre las opiniones que los maestros vertieron se encuentran: "Grandes potencias mundiales le dan más importancia a la educación artística", "No se le da el mismo tiempo y la importancia como a otras materias", "En comparación con las disciplinas básicas, se deja de lado esta área como materia extra" y "Se ve como una optativa". El investigador considera que los 11 profesores vieron en esta pregunta la oportunidad para compartir su percepción respecto a ser considerados como maestros de "segunda clase", dado que no imparten las materias consideradas como básicas, percepción que muchos otros maestros de artes en México comparten.

Otras categorías con menor representatividad fueron "Insuficiencia de tiempo para atender la cantidad de alumnos" (3 frecuencias) e "Insuficiencia de tiempo para alcanzar resultados o lograr productos" (2 frecuencias).

Solo tres profesores emitieron opiniones a favor del tiempo asignado a la materia de Artes. Sus opiniones fueron: "Las materias o asignaturas académicas requieren mucho tiempo del Currículo", "Con las sesiones que contamos los chicos mantienen interés en las actividades" y "Solo se trata de sensibilizar el gusto por el arte". Diez de los 103 maestros se abstuvieron de emitir sus opiniones. 


\begin{tabular}{|l|c|}
\hline \multicolumn{1}{|c|}{ Categoría } & Frecuencia \\
\hline $\begin{array}{l}\text { Insuficiencia de tiempo para cubrir de manera adecuada cada uno de los } \\
\text { temas del programa y llevar a cabo las actividades }\end{array}$ & 38 \\
\hline Porque es muy poco el tiempo (sin justificar) & 22 \\
\hline Insuficiencia para ejercer un impacto y beneficiar la vida del estudiante & 14 \\
\hline No se le da importancia & 11 \\
\hline Se abstuvieron & 10 \\
\hline Insuficiencia para atender la cantidad de alumnos & 3 \\
\hline Opiniones a favor & 3 \\
\hline $\begin{array}{l}\text { Insuficiencia para alcanzar resultados o lograr productos (festivales, } \\
\text { presentaciones) }\end{array}$ & 2 \\
\hline
\end{tabular}

Tabla 6. Opiniones sobre la cantidad de tiempo que se asigna a la clase de Artes.

\section{Conclusiones}

Los resultados del estudio indican que muchos de los maestros poseen una sólida formación disciplinar. Sin embargo, también se detectaron áreas de oportunidad significativas en la formación docente de muchos otros. Una cantidad importante de ellos carece de estudios profesionales. Incluso, hay maestros que solo cuentan con estudios de nivel secundaria. Por otro lado, muchos de ellos poseen una licenciatura que no está relacionada con la disciplina que imparten, por lo que no poseen una preparación especializada.

Podría decirse que, en lo referente a preparación práctica, los resultados sugieren que muchos de los maestros poseen por lo menos "suficientes" conocimientos y herramientas para llevar a cabo un buen trabajo. Sin embargo, cabe destacar que el poseer un conocimiento suficiente, aunque representa el mínimo para desempeñar la labor docente, no constituye el ideal en la preparación de un maestro (sobre todo a nivel de educación secundaria) sino que se debe procurar que los maestros tengan un conocimiento disciplinar sólido, que los habilite para resolver de manera efectiva los retos que implica la impartición de su asignatura.
En lo que se refiere a conocimientos teóricos que sustenten la práctica, existen áreas de oportunidad importantes. Salvo en el caso de los maestros de teatro (en que la mayoría son especialistas en esa disciplina) en general los resultados indican un desconocimiento importante de las propuestas metodológicas de pedagogos como Dalcroze, Orff, Kodaly, Lowenfeld, Laban y demás. Del mismo modo, se detectaron áreas de oportunidad en el conocimiento sobre los enfoques de educación artística ("la educación por el arte", "la educación a través del arte" "la educación en las artes"). Adicionalmente, se detectó falta de participación de los maestros en cursos de formación docente, talleres, seminarios y demás que podrían contribuir para que los maestros subsanen sus áreas de oportunidad.

Por otro lado, algunos maestros parecen desconocer la existencia de los materiales didácticos que, aunque escasos, pueden ser de utilidad para el maestro y la mayoría, indica estar en desacuerdo con las características de los programas de Artes (secundaria) y de educación artística (primaria). Por lo anterior, es de gran importancia que el IEA mantenga un diálogo abierto con sus maestros de educación artística, y favorezca la impar- tición de cursos de educación continua y talleres que permitan al maestro mantenerse actualizado. Por lo que respecta al descontento generalizado por el poco tiempo que se otorga a la asignatura y el poco apoyo que brindan las instituciones educativas, es importante que el maestro llegue a especializarse aún más para que pueda diseñar actividades mejor estructuradas a través de las cuales pueda llevar a cabo su labor docente de una manera eficiente, un aspecto a abordar a través de cursos de educación docente continua.

Finalmente, el número desigual de maestros que enseñan las distintas disciplinas tiene una grave implicación académica, ya que favorece que las materias se impartan indiscriminadamente, de tal manera que el estudiante no puede escoger que disciplina desea aprender. Así, puede darse que, debido a la distribución de las cargas académicas de los maestros, los estudiantes de un grupo deban tomar una de las disciplinas durante los tres años. Con lo anterior, se evita que el es tudiante entre en contacto con las otras disciplinas artísticas y pueda identificarse con ellas, e inclusive, desarrollar un desagrado hacia las artes. Implicaciones para la Educación Superior. 


\section{Implicaciones para la docencia y la educación superior}

Como puede observarse, existen debilidades importantes en la formación docente de los maestros de educación artística del IEA. Sin embargo, el autor ve en todas esas limitaciones, áreas de oportunidad importantes tanto para los maestros de educación artística a nivel básico como para los que se desempeñan a nivel superior. La experiencia que el autor ha tenido con las autoridades del Instituto de Educación de Aguascalientes (IEA) al llevar a cabo sus investigaciones, ha sido de gran apertura, consideración y deseo de cooperación. Por lo que respecta a la actitud de los maestros de educación artística a nivel preescolar, primaria y secundaria del IEA, ésta ha sido de puertas abiertas. Durante una serie de talleres de educación artística totalmente gratuitos impartidos por el investigador a los maestros de educación artística del IEA, pudo constatar su gran sensibilidad y entusiasmo, así como la disposición œ para aprender y el deseo de superación. $\stackrel{\sim}{\sim}$ Del mismo modo, durante las actividades Q llevadas a cabo en el taller, quedo de maป nifiesto la enorme creatividad, disciplina a y entrega de los docentes. Más aún, los ह่ mismos maestros han mostrado agrade- cimiento hacia la universidad por haberlos tomado en cuenta y han expresado su deseo de que ese tipo de experiencias y acercamiento entre la educación superior y la educación básica se siga dando.

Este universo de la educación artística a nivel básico en el estado de Aguascalientes (y en México) se vislumbra como una fuente de oportunidades para la investigación, la investigación aplicada, la difusión, la vinculación, el desarrollo de proyectos en conjunto, la creación de materiales didácticos, la implementación de talleres, cursos y diplomados y porque no, la creación de más licenciaturas especializadas en la pedagogía de las artes. Como lo destaca Errázuriz (2001, p. 12):

“Obviamente, para mejorar la calidad de la educación artística, es imprescindible la convicción y el perfeccionamiento del profesorado, porque sin su participación no habrá reformas educacionales. También es fundamental contar con autoridades visionarias que reconozcan el valioso aporte que pueden hacer las artes en la educación de la niñez y la juventud $y$, por lo tanto, que demuestren con hechos concretos su voluntad de implementar nuevas políticas educacionales tendientes a desarrollar la enseñanza de las Artes. De igual modo, sin la contribución de las instituciones que buscan apoyar".

Actualmente, el Instituto de Educación de Aguascalientes se enfrenta a un gran reto; la Secretaria de Educación Pública de México está exigiendo la profesionalización de todos los maestros del país por lo que se hace necesario tomar acciones para regularizar a los maestros que no cuenten con título profesional o por lo menos favorecer que se sigan capacitando y superando. En este rubro, las instituciones de educación superior podrían tomar este reto y proponer soluciones que se adecuen a las necesidades de los maestros.

Como puede verse, existe aquí un área de oportunidad muy importante en los ámbitos de la vinculación, la difusión, la docencia y la investigación, oportunidad que puede y debe ser atendida por los profesores-investigadores a nivel superior, pues son áreas sustantivas de la vida universitaria. Para suplir tales necesidades resulta congruente que las universi- dades y otras instituciones de educación superior deberían replantear sus licenciaturas en artes, contemplando que sean adecuadas a la misión de la Educación Artística en el siglo XXI.

Para finalizar, el autor considera que se deben reconocer los avances de la Secretaría de Educación Pública, en el ámbito de la educación artística y que las instituciones educativas a nivel superior, tomando en cuenta esos avances, deben promover la vinculación con el sector educativo básico y hacer propuestas que puedan redundar en beneficio de todos.

\section{Referencias}

Ávila, C. A. (2013). Acerca de la Expectativas Musicales en la Actualidad. Docere: Revista del Departamento de Formación y Actualización Académica. 8, 5-7.

Autor. (2016).

Creswell, J. W. (2001). Educational Re search:Planning, Conducting, and Evaluating Quantitative and Qualitative Research. Boston, MA: Pearson. 
Errázuriz, L. (2001). "Mejorar la calidad de la educación artística en América Latina: un derecho y un desafío", en Métodos, contenidos y enseñanza de las artes en América Latina y el Caribe. Conclusiones de la Conferencia Regional sobre Educación Artística en América Latina y el Caribe, 6 al 19 de octubre, Uberaba, Brasil. Recuperado de http://repositorio.minedu.gob.pe/bitstream/handle/MINEDU/5160/M\%C3\%A9todos\%2C\%20contenidos\%20y\%20ense $\%$ C3\%B1 anza\%20de\%20las\%20artes\%20 en $\% 20$ Latinoam $\%$ C3\%A9rica $\% 20 y \% 20$ el\%20Caribe $\% 20 \% 201$ a $\% 20$ educaci\%C3\%B3n\%20art\%C3\%ADstica\%20y\%20la\%20creatividad\%20en\%20la\%20escuela\%20 primaria\%20y\%20secundaria.pdf?sequence $=1$ \&isAllowed $=y$

Giráldez, A. (2011). Fundamentos metodológicos de la educación artística. En L. Jiménez, I. Aguirre y L. G. Pimentel (Eds.), Educación Artística, Cultura y Ciudadanía (pp. 89-96). Madrid, España: Organización de Estados Iberoamericanos para la Educación, la Ciencia y la Cultura (OEI).

González, M. L. (1999). La sistematización y el análisis de los datos cualitativos. En: Mejía, A. R. y Sandoval, A. S. (coord.), Tras las vetas de la investigación cualitativa (pp. 157-173). México: ITESO.

Harwell, S. (2003). Teacher Professional Development: It's Not an Event, It's a Process. CORD. http://www.cord.org/uploadedfiles/HarwellPaper.pdf

Martínez, M. C. (2010). Docentes de educación artística. Experiencias en el marco de la formación continua. Revista Iberoamericana de Educación. 52, 95-108.

Pimentel, L. G. Coutinho, R. G. Y Guimarães, L. (2011). La formación de profesores de arte: prácticas docentes. En L. Jiménez, I. Aguirre y L. G. Pimentel (Eds.), Educación Artística, Cultura y Ciudadanía (pp. 115-122). Madrid, España: Organización de Estados Iberoamericanos para la Educación, la Ciencia y la Cultura (OEI).

SEP. (2006a). Plan de Estudios 2006. Educación Básica Secundaria. Recuperado de: http://www.sev.gob.mx/servicios/rvoe/2010/sec_gral/normatividad_secgral/plan_estudio_secgral.pdf

30 Educación y Pedagogía
SEP. (2006b). Artes. Guía de trabajo. Primer Taller de Actualización sobre los Programas de Estudio 2006 (2006). Recuperado de https://coleccion.siaeducacion.org/sites/default/files/primer_taller_guias_danza.pdf

SEP. (2009). Curso: Introducción a la educación Artística en el Contexto Escolar. Recuperado de http://www.centrodemaestros.mx/enams/LAEDUCACIONARTISTICA.pdf

SEP. (2011a). Plan de Estudios 2011. Recuperado de http://www.excelduc.org.mx/ sys-uploads/documentos/plan_de_estudios_2011_educacion_basica.pdf

SEP. (2011b). Programas de Estudio 2011, Guía Para el Maestro, Educación Básica Se cundaria, Artes, 2011. Recuperado de: http://www.centrodemaestros.mx/programas estudio/Artes_SEC.pdf

SEP. (2011c). Las artes y su enseñanza en la educación básica. Recuperado de: https:// rarchivoszona33.files.wordpress.com/2012/08/75388177-las-artes-y-su-ensenanzaen-la-educacion-basica.pdf

SEP. (2014). Perfil, Parámetros e Indicadores para Docentes y Técnicos Docentes y Propuesta de etapas, aspectos, métodos e instrumentos de evaluación, para el año escolar 2014-2015. Secretaria de Educación Pública. Recuperado de http://servicioprofesionaldocente.sep.gob.mx/content/ba/docs/parametros_indicadores/Completo.pdf

Shulman, L. S. (2005). Conocimiento y enseñanza: fundamentos de la nueva reforma. Profesorado. Revista de currículum y formación del profesorado, 9(2), 1-30.

Touriñán, J. M. (2006). Educación en valores y experiencia axiológica: el sentido patrimonial de la educación. Revista Española de Pedagogía, 234, 227-248.

Yohary, A. B. Y Araujo, C. (2014). Retos de la educación artística en el siglo XXI en México. Universita Ciencia. Revista Electrónica de Investigación de la Universidad de Xalapa, 3(8), 59-69. 